\title{
An examination of the wind-driven effect on the drift of precipitation particles using the Chung-Li VHF radar
}

\author{
Yen-Hsyang Chu \\ Institute of Space Science, National Central University, Chung-Li, Taiwan \\ Tsai-Yuan Chen \\ Institute of Electronics, National Chiao Tung University, Hsin-Chu, Taiwan \\ Tszer-Hong Lin \\ Institute of Space Science, National Central University, Chung-Li, Taiwan
}

\begin{abstract}
One of the essential assumptions made in estimating the terminal velocity and the drop-size distribution of hydrometeor from the precipitation Doppler spectrum observed by VHF/UHF radar (or wind profiler) is that all of the precipitation particles are moving together at the same velocity as the ambient wind. However, this may not be the case. In this paper, taking advantage of the capability of simultaneously measuring the motions of precipitation particles and the clear-air turbulence with a VHF radar, we develop a method to examine the wind-driven effect on the drift of precipitation particles using the Chung-Li VHF radar. By appropriately setting the radar parameters and steering the radar beams, experimental results show that the drift velocity of the precipitation particles in response to the drag force exerted by the background atmosphere is not the same as the ambient wind velocity, approximately $83 \%$ of the latter in this case. This feature indicates that the precipitation particles are not moving with the background wind, causing inaccurate estimations of the terminal velocity and the drop-size distribution of hydrometeor if this effect is not taken into account.
\end{abstract}

\section{Introduction}

It is well recognized that a radar operating at VHF band not only can detect the echoes from atmospheric refractivity fluctuations, but also can be used for the observation of precipitation. Because of this merit, several important precipitation-related parameters and features which cannot be provided directly by conventional microwave meteorological radar, such as the true terminal velocity of hydrometeor, size distributions of liquid and solid precipitation particles, vertical air motion associated with intense precipitation, interaction between hydrometeor and the ambient atmosphere, and so on, can be observed using VHF radar if the experiment is set up appropriately [Wakasugi et al., 1986; Rajopadhyaya et al., 1993; Chu et al., 1991; Chu and Lin, 1994].

Because most of the desired information is imbedded in it, the Doppler spectrum of VHF radar returns

Copyright 1997 by the American Geophysical Union.

Paper number 97RS00057.

0048-6604/97/97RS-00057\$11.00 is usually calculated to estimate the precipitationrelated parameters. In light of the fact that the falling of the precipitation particles in the cloud will be affected inevitably by the motion of the background atmosphere, the Doppler spectrum from precipitation will include the contributions of ambient wind and turbulence. Under this consideration, the contribution of the background atmosphere to the observed Doppler spectrum of precipitation should be removed in deriving the drop-size distribution from the precipitation Doppler spectral width. In general, the mathematical relation of the Doppler spectra between precipitation particles and refractivity fluctuations for a vertically pointed radar beam can be formulated as [Wakasugi et al., 1986]

$$
S_{r}(\omega)=S_{t}(\omega) \otimes S_{p}\left(\omega-\omega_{o}\right)
$$

where $S_{r}(\omega)$ is the observed precipitation Doppler spectrum, $S_{t}(\omega)$ represents the Doppler spectrum of refractivity fluctuations, and $S_{p}\left(\omega-\omega_{o}\right)$ is the size distribution of precipitation particles in the Doppler spectral domain centered at Doppler frequency $\omega_{o}$, and $\otimes$ represents the convolution operator. Note that 
the shape of $S_{t}(\omega)$ is usually assumed to be Gaussian because of the beam broadening and turbulent broadening effects [Woodman and Chu, 1989]. Superficially, the shape of $S_{p}\left(\omega-\omega_{o}\right)$ is not Gaussian due to the exponential form or Gamma pattern of the drop-size distribution [Marshall and Palmer, 1948; Ulbrich, 1983]. However, because the radar echo power from precipitation is proportional to the 6th power of the diameter of the precipitation particle, the pattern of $S_{p}\left(\omega-\omega_{o}\right)$ will be quasi-Gaussian in the Doppler spectral domain [Atlas et al., 1973]. Consequently, for the sake of mathematical simplicity, the shape of $S_{p}\left(\omega-\omega_{o}\right)$ can be treated with Gaussian form, causing the reasonable approximation of Gaussian pattern to $S_{r}(\omega)$. In this case, the information of precipitation particles can be separated from $S_{r}(\omega)$, which is contaminated by $S_{t}(\omega)$, through the following relation:

$$
\sigma_{p}^{2}=\sigma_{r}^{2}-\sigma_{t}^{2}
$$

where $\sigma_{r}^{2}, \sigma_{p}^{2}$, and $\sigma_{t}^{2}$ are the variances of $S_{r}(\omega)$, $S_{p}\left(\omega-\omega_{o}\right)$, and $S_{t}(\omega)$, respectively. Once $\sigma_{p}^{2}$ is estimated, in association with the observed radar reflectivity and the terminal velocity of hydrometeor, the parameters related to the shape of the drop-size distribution can thus be obtained, provided the specific relation between drop size and fall speed and the shape of the size spectrum of hydrometeor are assumed [Atlas et al., 1973]. Recently, several techniques were employed to retrieve the drop-size distribution from observed precipitation Doppler spectra. Wakasugi et al. [1986] fitted a spectrum based on the Marshall-Palmer distribution to the observed spectra by a least squares method. Currier et al. [1992] fitted a theoretical Doppler spectrum based on a Gamma function form of drop-size distribution to the observed spectra by using a nonlinear least squares routine. Rajopadhyaya et al. [1993] employed the methods of deconvolution without assuming a specific shape of drop-size distribution to estimate the dropsize distribution from the Doppler spectra measured by wind profiler radars. However, no matter what technique is employed in the estimation of the dropsize distribution, the mathematical expression connecting the motions of the background atmosphere and the hydrometeor is based on (1).

It is worth pointing out that in applying (1) to deduce the size distribution of precipitation particles, several important assumptions are made implicitly: (1) The Gaussian Doppler spectral shape of $S_{t}(\omega)$ is not distorted by the vertical air motion which always exists in the cloud with precipitation; (2) the shape of precipitation particles is spherical, i.e., the spectral shape of $S_{r}(\omega)$ is not influenced by the spectral narrowing effect of the aspect sensitivity, which originated from the anisotropic characteristics of the radar target; (3) the spatial distribution of the precipitation particles is uniform in the scattering volume; (4) the ambient wind velocity and the hydrometeor size distribution, which play crucial roles in determining the radar reflectivity, mean Doppler shift, and the spectral width of the precipitation Doppler spectrum, are both constant during the period of radar signal processing, namely, the time interval for coherent and incoherent integrations; and (5) the precipitation particles are assumed to move with the ambient wind at the same velocity so that the effects of the beam broadening and the turbulent broadening on $S_{t}(\omega)$ can be thought to be identical to those on $S_{r}(\omega)$. This frozen-in assumption is the most important one in removing the contribution of the background atmosphere from the precipitation Doppler spectrum using the techniques mentioned in the previous paragraph. Assumption 1 is usually true because the atmospheric vertical velocity is seldom greater than the horizontal velocity, except for cases of intense updraft and downdraft in an extremely active convective cloud. However, if the vertical velocity is considerably greater than the horizontal wind speed, it can be shown that the shape of $S_{t}(\omega)$ obtained by a vertically pointed radar beam will be approximate to the exponential form rather than the Gaussian form. The discussion on the distortion effect of vertical air motion on the Doppler spectral shape will be presented in a future paper. Assumption 2 is basically true for liquid hydrometeor if the drop size is not sufficiently large [Battan, 1973]. However, observation shows that the VHF echo power from solid hydrometeor, that is, the snowflake, ice sheet, etc., is highly aspect sensitive, implying that the narrowing effect of the aspect sensitivity on the precipitation Doppler spectral shape should be taken into account in estimating the ice size distribution using a VHF radar [Woodman and Chu, 1989; Chu et al., 1991]. Assumptions 3 and 4 generally hold for the precipitating environment with stable wind and persistent precipitation in the widespread stratiform cloud. They are questionable, however, for a localized and short-lived convective cloud with variable wind and intermittent precipitation. Moreover, recent observational results obtained using the Chung-Li VHF radar show that 
even for the widespread frontal precipitation, the hydrometeors may distribute inhomogeneously in the scatter volume with horizontal scale of about $500 \mathrm{~m}$ [Sue, 1996]. Therefore great caution should be taken in the use of (1) to deduce the size distribution of the precipitation particle, especially for the precipitation in an active convective cloud. Assumption 5 plays a vital role in utilizing VHF/UHF radar to measure the precipitation-related parameters and phenomena. If this assumption is not valid, the results of estimating a precipitation particle size distribution made by VHF/UHF radar with the existing techniques as mentioned above will be doubtful. This is because in this case the clear-air component in the observed precipitation Doppler spectrum, which is related to the motions of background atmosphere through the beam broadening and turbulent broadening effects, will be not identical to the observed Doppler spectrum generated directly by the refractivity fluctuations, causing the problem of removing the influence of background atmosphere from the precipitation Doppler spectrum. Moreover, this assumption is also an essential one for a conventional microwave meteorological radar employed to estimate the ambient wind velocity from the precipitation echoes using the velocity-azimuth display (VAD) technique [Battan, 1973]. Because of its importance, it is worth examining the frozen-in assumption of hydrometeor so that the accuracy of the measurements of the wind and precipitation using a VHF/UHF and/or microwave meteorological radar can be improved.

With the capability of simultaneously observing the characteristics of precipitation and clear air of a VHF/UHF radar (or wind profiler), a method of examining the wind-driven property of hydrometeor is proposed in this article. After conducting an appropriate experiment at the Chung-Li VHF radar, we will present the observational evidence showing that the precipitation particles are not necessarily moving with the background wind at the same velocity. In section 2 , a theoretical consideration of dealing with the examination of the so-called frozen-in property of the precipitation particles in the ambient atmosphere will be introduced. An experimental setup at the Chung-Li VHF radar will be described in detail in section 3 . The observational results and discussion will be presented in section 4 . The conclusion will be given in section 5 .

\section{Theoretical Considerations}

When a precipitation particle is falling in the atmosphere, the motion of the ambient air and the size of the hydrometeor will determine its falling velocity. Assume that two radar beams, one pointed vertically and the other steered obliquely at the zenith angle of $\theta$, are employed simultaneously to observed the falling velocity of the precipitation particle. If the hydrometeor subjected to a drag force exerted by the ambient air flow is moving with the background wind at the same velocity, its Doppler frequency shift observed by an oblique radar beam is related to the Doppler frequency shifts of ambient wind and the terminal velocity of precipitation particles as

$$
f_{o}=\left(f_{t}-f_{a}\right) \times \cos \theta-f_{u}
$$

where $f_{o}$ (equal to $-2 \mathrm{~V}_{\mathrm{obs}} / \lambda$ ) is the Doppler frequency shift of the precipitation particle measured by the oblique radar beam, $f_{t}$ (equal to $-2 \mathrm{~W}_{t} / \lambda$ ) is the vertical Doppler frequency shift of the precipitation particle at the terminal velocity $\mathrm{W}_{t}, f_{a}$ (equal to $\left.-2 \mathrm{~W}_{a} / \lambda\right)$ is the vertical Doppler frequency shift of the ambient air at the vertical velocity $\mathrm{W}_{a}$, and $f_{u}$ (equal to $-2 \mathrm{U} \sin \theta / \lambda$ ) is the oblique Doppler frequency shift corresponding to the horizontal component $U$ of the ambient air velocity in the pointing direction of the oblique beam. The geometry related to the parameters $\mathrm{W}_{t}, \mathrm{~W}_{a}$ and $\mathrm{U}$ for equation (3) are shown in Figure 1, where the vectors pointing toward (away from) the radar are responsible for the positive (negative) Doppler frequency shifts of the radar echoes. Although the ambient wind exerting a drag force on the precipitation particle compels it to move with the background air flow, the drift velocity of the hydrometeor may not necessarily be equal to that of the ambient wind. Notice that the drag force exerted on the precipitation particle by atmospheric flow is not only affected by the characteristics of the ambient atmosphere (e.g., temperature and density), but also by the functions of the shape and diameter of the hydrometeor. Moreover, the other characteristics of the hydrometeor itself, namely, the electricity property, dynamic behavior (vibration, rotation, and coalescence), thermal processes (evaporation and condensation of water vapor), and the small-scale turbulence around the hydrometeor during its falling, may presumably also influence the drag force on a precipitation particle exerted by the ambient atmosphere. Under these considerations it appears that the precipitation particle may not drift with the 


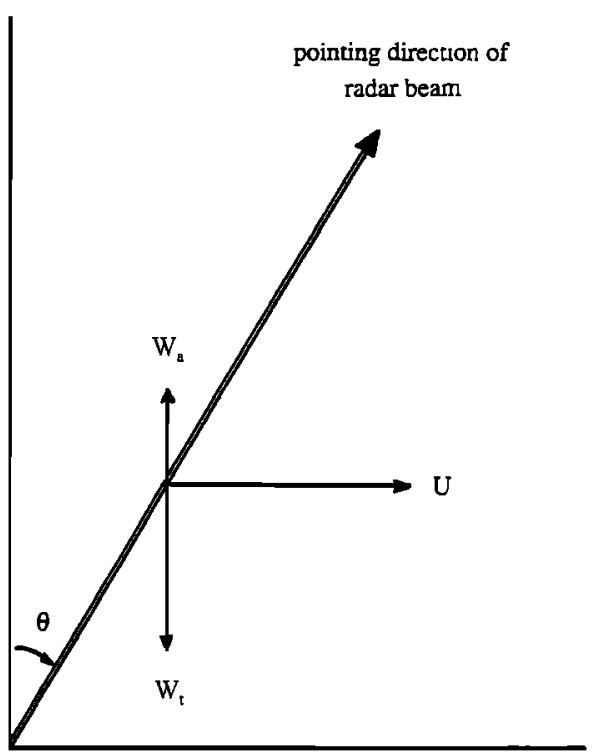

Figure 1. A schematic plot showing the geometrical relation between terminal velocity $\mathrm{W}_{t}$ of precipitation particles, vertical air velocity $\mathrm{W}_{a}$, and the horizontal wind speed $\mathrm{U}$ in the pointing direction of the oblique radar beam.

ambient wind. If this is the case, by introducing a fractional coefficient $\alpha$ to represent the imperfect drive of the background air flow on the drift of precipitation particles, (3) can thus be rewritten as

$$
f_{\alpha}=\left(f_{t}-\alpha f_{a}\right) \times \cos \theta-\alpha f_{u}
$$

where the assumptions that precipitation particles distribute uniformly in the radar probing region and that $\alpha$ is a linear and isotropic scalar constant have been made implicitly in (4). It is easy to realize from (3) and (4) that $\alpha$ is just the ratio of the hydrometeor drift velocity in response to the drag force exerted by the background atmospheric flow to the ambient wind velocity. Therefore $\alpha\left(\mathrm{W}_{a} \cos \theta+\mathrm{U} \sin \theta\right)$ represents the effective radial velocity component of the precipitation particle driven by the background atmosphere, which can be obtained from the clear-air Doppler spectra observed simultaneously by the vertical and oblique radar beams. From (4) it is apparent that in the case of $\alpha=1$, the hydrometeor will drift perfectly with (or be frozen in) the background air flow, while in the extreme case of $\alpha=0$, the hydrometeor will make no response to the drag of ambient wind. From (3) and (4) it is easy to obtain

$$
f_{o}-f_{\alpha}=(1-\alpha) \times f_{a r}
$$

where

$$
f_{a r}=-f_{a} \times \cos \theta-f_{u}
$$

Notice that $f_{a r}$ (equal to $-2 \mathrm{~V}_{r} / \lambda$ ) is the oblique Doppler frequency shift of the air having the radial velocity along the pointing direction of the oblique beam. In the case of Figure 1, because the plus and minus signs of the Doppler frequency shifts have been considered in the equations and $0<\alpha<1$ in definition, it is obvious that $f_{o}-f_{\alpha}$ (equal to $\Delta f_{x}$ ) $<$ 0 . Similarly, if the radar beam is steered in the opposite direction and we assume that the precipitation and the clear-air conditions are the same as those shown in Figure 1, it is easy to have

$$
f_{o}-f_{\alpha}=(1-\alpha) \times f_{a r}
$$

where

$$
f_{a r}=-f_{a} \times \cos \theta+f_{u}
$$

In this case, $f_{o}-f_{\alpha}$ (equal to $\Delta f_{y}$ ) $>0$ and $\left|\Delta f_{x}\right|>$ $\left|\Delta f_{y}\right|$, provided $f_{a} \times \cos \theta<f_{u}$. Therefore it is obvious that the wind-driven characteristics of hydrometeors can be examined if the systematic features, that is, $\Delta f_{x}<0$ and $\left|\Delta f_{x}\right|>\left|\Delta f_{y}\right|$, are always observed by steering radar beams toward different off-zenith directions. In addition to the discrepancy between $f_{o}$ and $f_{\alpha}$, the ratio of $f_{\alpha}$ to $f_{o}$ (defined as $R_{f}$ ) can also be employed to examine the wind-driven property of hydrometeor. If $R_{f}$ is equal to unity, it means the hydrometeors are perfectly drifting with background air flow and $\alpha=1$. If the value of $R_{f}$ deviates from unity, the horizontal velocity of precipitation particles pushed by the ambient wind will be different from that of the ambient atmosphere. The problem remains of how to calculate the value of $\alpha$ from the information of the observed Doppler frequency shifts of wind and precipitation. Because the Doppler frequency shift of the precipitation particles observed by the vertical radar beam, $f_{v}$ (equal to $\left.-2 W_{\text {obs }} / \lambda\right)$ can be expressed as

$$
f_{\nu}=f_{t}-\alpha f_{a}
$$

Substituting (9) into (4), we have

$$
\alpha=\left(f_{v} \times \cos \theta-f_{\alpha}\right) / f_{u}
$$

where $f_{u}$ can be obtained from the observed values of $f_{a}$ and $f_{a r}$ in accordance with (5) or (8), depending on the geometry of the radar beam pointed. Therefore, according to the simultaneous measurements of the 
Doppler frequency shifts of precipitation particles and the ambient winds from two radar beams at different pointing direction, the frozen-in property of the precipitation particles can be examined.

\section{Experiment Setup and Data Analysis}

\subsection{Chung-Li VHF Radar}

The Chung-Li VHF radar consists of three independent and identical modules, and each of the modules contains its own transmitter, receiver, antenna array, signal preprocessor, and other essential system units. This radar is located on the campus of National Central University in Taiwan. The operational frequency of the Chung-Li VHF radar is $\mathbf{5 2}$ $\mathrm{MHz}$ (corresponding to 5.77-m wavelength), and the peak transmitted power is $180 \mathrm{~kW}(3 \times 60)$. The maximum duty cycle is $2 \%$, and the pulse width can be set arbitrarily from $1 \mu \mathrm{s}$ to $999 \mu \mathrm{s}$. However, only five receiver bandwidths can be chosen on the Chung-Li VHF radar, namely, $1,0.5,0.25$, and $0.125 \mathrm{MHz}$ and $62.5 \mathrm{KHz}$. Two phase-coding schemes are implemented on the Chung-Li VHF radar for different purposes of the experiment. One is the complementary code, with adjustable code lengths of $2,4,8$, and 16 elements. The other one is the Barker code, with adjustable code lengths of 7, 9, and 13 elements. The antenna array of the Chung-Li VHF radar used for mesosphere-stratosphere-troposphere (MST) experiments consists of three square antenna modules with 64 Yagi antenna elements $(8 \times 8)$ each. The whole antenna array is arranged as a triangle with side lengths of 45,45 , and $40 \mathrm{~m}$. Except for the vertical direction, the boresight of the antenna beam for each module can also be pointed obliquely toward north, west, south, and east with a fixed $17^{\circ}$ zenith angle. Note that the azimuthal direction that the antenna beam is steered in is not due geographical north, west, south, and east, but has a counterclockwise $22.3^{\circ}$ azimuthal angular difference from the respective due geographical direction. The total physical antenna area is $4800 \mathrm{~m}^{2}(3 \times 40 \times 40)$. The main antenna beam for each module has a half-power beam width of $7.4^{\circ}$. For more information on the characteristics of the Chung-Li VHF radar, see Rottger et al. [1990].

\subsection{Experiment Setup}

As described in the previous section, at least two radar beams, one at vertical incidence and the other pointed obliquely, are required to operate simultaneously so that the experiment of examining the wind-driven property of precipitation particles can be carried out. Alternatively, if the temporal variations in ambient wind and precipitation can be ignored during a short period, it is not necessary to operate the vertical and oblique beams simultaneously in the examination of the frozen-in characteristics of the precipitation particles. In this case, the experimental scheme that one of the two radar beams be steered toward vertical (or oblique) direction subsequent to the other beam being steered obliquely (or vertically) is permissible, as long as the total observation period of these two radar beams is short enough. However, how short is short enough for the observation period in which the wind and precipitation can be treated to be constant in examining the frozen-in behavior of precipitation particles is still not well-documented. Under this consideration, in order to avoid the possible bias due to the temporal variations in wind and precipitation, the vertical and oblique radar beams are operated simultaneously in the experiment of examining the frozen-in characteristics of hydrometeor conducted at the Chung-Li VHF radar.

The precipitation data employed for this experiment were taken on April 13, 1996, 0437-0540 LT by the Chung-Li VHF radar. During this period a cold front was passing through Taiwan island from southwest toward northeast. An examination of the rainfall record measured by the meteorological station located on the campus of the National Central University shows that the total amount of rainfall corresponding to this cold front from 0800 to $2000 \mathrm{LT}$ is about $58 \mathrm{~mm}$. Three radar beams were operated simultaneously. Two of them were always pointed vertically during the period of the experiment, and the other one was steered obliquely toward north, east, south, and west in sequence. The observational duration for each direction is $34.56 \mathrm{~s}$. The main radar parameters were set as follows: pulse length of $2 \mu \mathrm{s}$, interpulse period of $300 \mu \mathrm{s}$, and coherent integration time of $0.09 \mathrm{~s} ; 40$ range gates were recorded.

\subsection{Data Analysis}

When the complex raw radar echoes were recorded in the data storage unit, the Doppler spectra are calculated by using a 64-point fast Fourier transform (FFT) algorithm on the radar returns. Six raw spectra are averaged incoherently to produce an average spectrum for further analysis. In view of the complexity of the observed Doppler spectrum, the identification of the spectral components for precipitation and refractivity can only be done manually. Once they are separated, the echo power, mean Doppler frequency 
shift, and spectral width for these two spectral components are estimated with the least squares method, in which the Gaussian curve is employed to best fit the corresponding Doppler spectral component.

From the theoretical equations presented in the previous section, the falling velocities of precipitation particles observed by vertical and oblique radar beams will play a crucial role in examining the frozen-in characteristics of the hydrometeors in the ambient air flow. In order to estimate the diameter of the hydrometeor from its fall speed, the following expression is employed extensively [Atlas et al., 1973]:

$$
v_{d}=9.65-10.3 \exp (-6 D)
$$

where $v_{d}$ (in units of meters per second) is the fall speed of hydrometeor with the diameter $D$ (in units of centimeters) with respect to the still air. However, when precipitation particles are falling in the atmosphere, their fall speed will be affected by the background air density through the viscous drag exerted by the air. Because (11) is applicable only near the Earth's surface, a necessary correction factor should be given on the right side of the equation if this equation is applied to other altitudes for the estimation of the fall speed of hydrometeor. Although a number of different correction factors have been introduced by many investigators [Lin et al., 1983; Beard and Heymsfield, 1988; Zrnic et al., 1993], the correction factor employed in this study will be $\left(\rho_{0} / \rho\right)^{0.4}$ [Foote and du Toit, 1969; Atlas et al., 1973], where $\rho_{0}$ and $\rho$ are the air density at the ground and the level of observation, respectively.

\section{Observations and Discussion}

As mentioned earlier, the vertical and oblique antenna beams are operated simultaneously in this experiment. Figure 2 presents an example of the Doppler spectra observed by vertical (upper panels) and northward (lower panels) beams at heights from 2.4 to $3.3 \mathrm{~km}$. It is obvious from Figure 2 that the refractivity fluctuation echoes and the precipitation echoes occurring simultaneously in the vertical and oblique radar beams can be discerned unambiguously in each panel, where the broad spectral peaks located in the spectral domain with larger positive frequency shifts are the precipitation echoes, while the other spectral peaks with stronger echo intensities and narrow widths correspond to the radar returns of refractivity fluctuations. It is noteworthy that although the vertical and oblique beams are operated simultaneously to observe precipitation in this experiment, in numerous

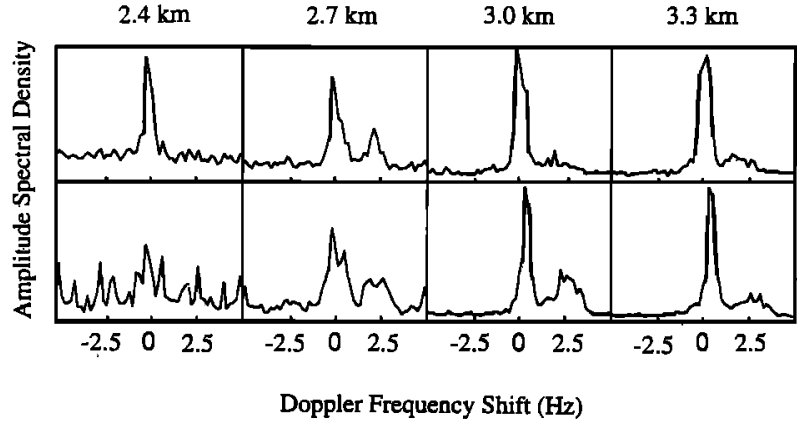

Figure 2. Vertical (upper panels) and northward (lower panels) Doppler spectra observed simultaneously on April 13,1996 , at around $1638 \mathrm{LT}$ with the incoherent integration time of $34.56 \mathrm{~s}$.

cases, only one radar beam, either vertical or oblique, can detect the precipitation echoes, no matter what altitude at which the scattering volume is situated. Because the zenith angle of the oblique beam is $17^{\circ}$ and because most precipitation occurs below the height of 4 $\mathrm{km}$ (as shown later), the horizontal separation of the boresight between vertical and oblique beams at $4 \mathrm{~km}$ is about $1.2 \mathrm{~km}$. Because the antenna beam width of the Chung-Li radar is $7.4^{\circ}$, it is easy to calculate that the horizontal distance between the vertical and oblique beams at a height of $4 \mathrm{~km}$ without overlapping is about $700 \mathrm{~m}$ and is about $500 \mathrm{~m}$ at the height of $3 \mathrm{~km}$. Therefore the feature of the precipitation echoes that can only be detected by one of two simultaneous radar beams at different pointing directions strongly implies that the precipitation particles distribute inhomogeneously in the region with a horizontal dimension of around $500-700 \mathrm{~m}$, causing difficulty in examining the wind-driven effect on the hydrometeors.

Figure 3 is the height-time distribution of the horizontal wind velocity deduced from the clear-air Doppler spectra. As indicated, the northwest wind dominates the bulk motion of the ambient atmosphere during the period of experiment. Because the sign and the magnitude of $f_{o}$ (or $f_{\alpha}$ ) are largely determined by the direction of radial velocities of background wind, it can be expected that $f_{o}-f_{\alpha}(=$ $\Delta f$ ) obtained by the eastward beam and the southward beam will be in opposition to those observed by the northward and westward beams, provided $\alpha \neq 1$. These observational results will be presented later.

Figure 4 is the height-time variation of the hydrometeor falling velocity deduced from the precipitation Doppler spectra observed by the vertical beam. It is clear from Figure 4 that the falling velocities of the 


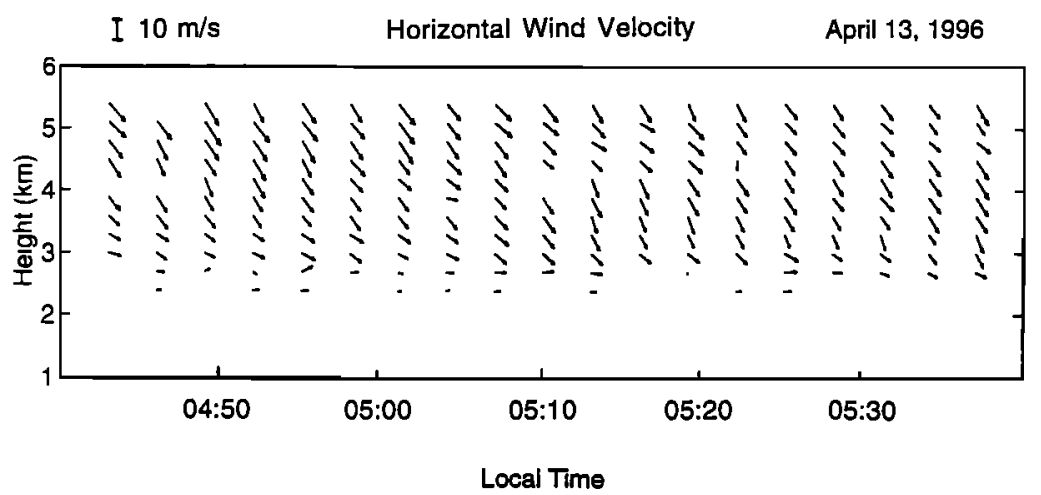

Figure 3. Height-time distribution of the horizontal wind velocity deduced from the mean Doppler shifts of northward and eastward radar spectra, where the contribution of vertical wind has been removed.

precipitation particles range from about $2 \mathrm{~m} / \mathrm{s}$ at 4.2 $\mathrm{km}$ to about $6 \mathrm{~m} / \mathrm{s}$ at $2.4 \mathrm{~km}$. An abrupt change in falling velocity is seen in the height range from 3.6 to $4.2 \mathrm{~km}$. Examining the temperature profile observed at Pan-Chiao rawinsonde station, about $25 \mathrm{~km}$ northeast of the Chung-Li radar station, demonstrates that the altitude of $0^{\circ} \mathrm{C}$ isothermal is $3.85 \mathrm{~km}$. Therefore the sudden increase in the falling speed of hydro- meteors shown in Figure 4, which is formed owing to the coalescence of the melting precipitation particles in the melting layer [Rogers, 1976; Chu et al., 1991], is consistent with the temperature profile observed by the rawinsonde. Note also that the precipitation occurring mainly in the height range from 2 to $4.5 \mathrm{~km}$ distributes unevenly with height and time. As mentioned before, this feature implies that the spatial

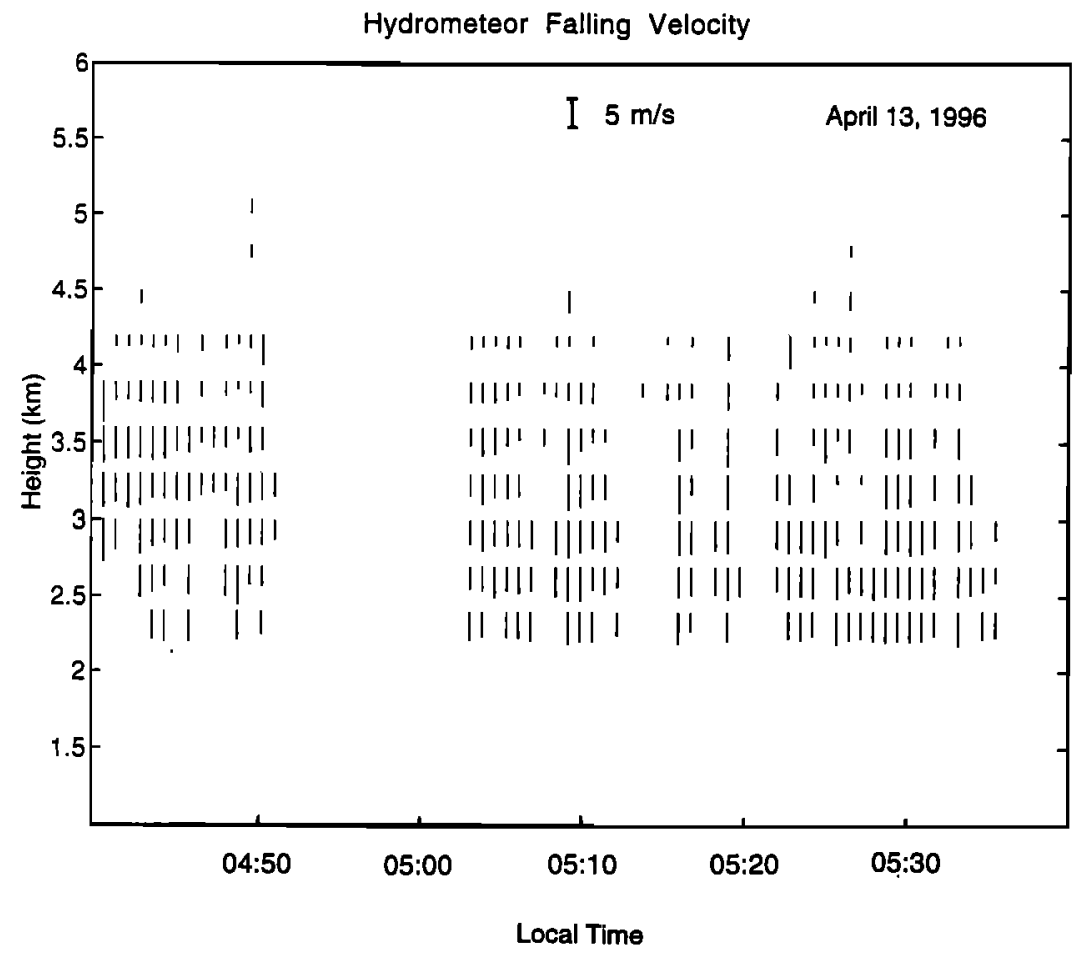

Figure 4. Height-time variation of the hydrometeor falling velocity deduced from the Doppler spectra observed by the vertical radar beam. 


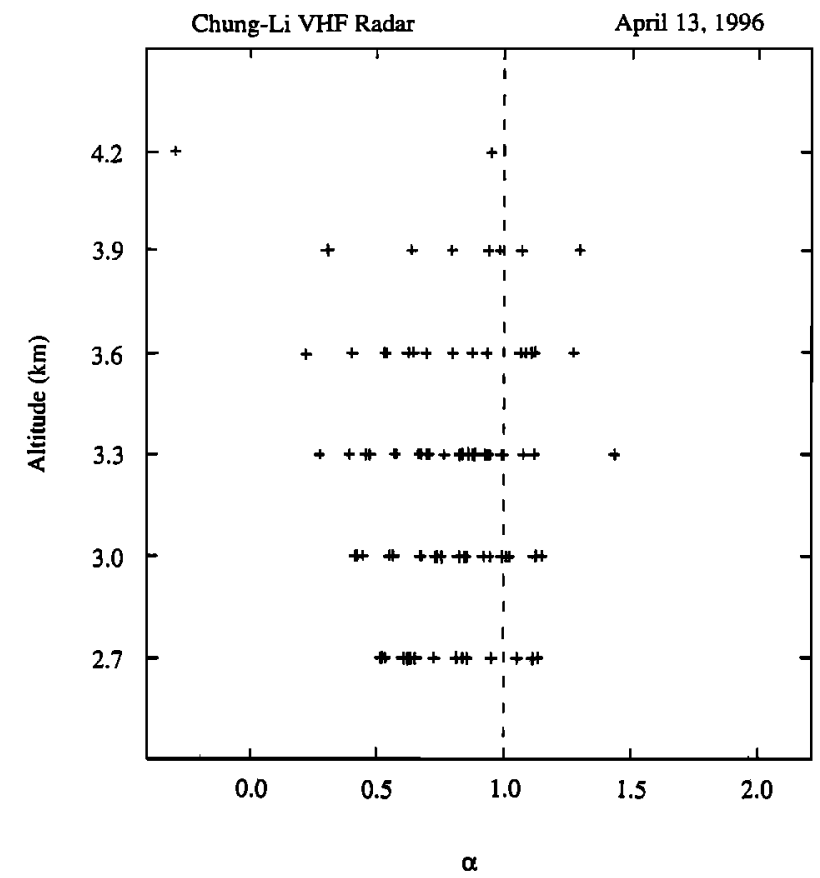

Figure 5. Height distribution of $\alpha$ values calculated from equation (10).

distribution of precipitation may be inhomogeneous, causing the biased estimation of the value of $\alpha$. This problem will be discussed in more detail later.

Once the observed Doppler frequency shifts of precipitation and the refractivity fluctuations measured with vertical and oblique beams are obtained, the examination of the wind-driven property of precipitation particles can be carried out. Figure 5 presents the height distribution of $\alpha$ values calculated from (10). According to the definition of $\alpha$, if the precipitation particles are frozen in the background atmosphere, it can be expected that the magnitude of $\alpha$ will be identical to unity. However, Figure 5 shows that, in general, the observed $\alpha$ values are located systematically on the left side of the line corresponding to the value of $\alpha=1$. Computation denotes that the averaged magnitude of $\alpha$ is 0.83 , indicating that the efficiency of the background wind driving the precipitation particles to move with ambient air is, on average, 0.83 . This observational evidence raises an important problem regarding the validation of the existing methods employed to estimate the drop-size distribution and the terminal velocity of the precipitation particles from Doppler spectra observed by VHF/UHF radar (or wind profiler). As mentioned in section 1, one of the basic assumptions made in applying (1) to deduce the size distribution of precip- itation particles is that all of the hydrometeors are moving perfectly with background wind such that the beam broadening and turbulent broadening effects contributed by ambient atmosphere on the observed Doppler spectral width of precipitation can be removed totally. Similarly, under the same assumption the exact terminal velocity of precipitation particles can be obtained by subtracting the contribution of the vertical wind velocity from the observed precipitation falling velocity. However, if this crucial assumption is not valid, the results of applying current methods to deduce the terminal velocity and the drop-size distribution of precipitation particles from the radar returns of VHF/UHF wind profiler radar will be questioned. Note that the magnitude of $\alpha$ represents the response of the drift of the hydrometeor to the drag of ambient wind. From Figure 5 it is obvious that, on average, the effective horizontal drift velocity of the precipitation particles will be $83 \%$ of the ambient wind velocity. In view of the fact that the beam broadening spectral width is proportional to the horizontal drift velocity of the targets in the radar volume, the beam broadening spectral width contributing to the observed Doppler spectral width of precipitation will be $83 \%$ smaller than that of the background atmospheric refractivity fluctuations. Therefore the estimations of the drop-size distribution and the terminal velocity of precipitation particles will be inaccurate if the conventional method is employed and the effect of imperfect drift of precipitation particles with the background wind is not taken into account.

Figure 5 also shows that a number of data points have values unrealistically greater than 1 or smaller than 0 . Several data points with large scatter result from the statistical uncertainties associated with the fluctuations of the radial velocities of the precipitation and clear-air turbulence estimated from the observed Doppler spectra. Calculation shows that a $10 \%$ variation in radial wind speed and Doppler velocity of precipitation particles deduced from the observed Doppler spectra will result in a $58.8 \%$ variation in $\alpha$, provided the averaged magnitudes of $\alpha$-related parameters are given as $\langle\mathbf{U}\rangle=10 \mathrm{~m} / \mathrm{s},\left\langle\mathrm{W}_{a}\right\rangle$ $=1 \mathrm{~m} / \mathrm{s},\left\langle\mathrm{W}_{t}\right\rangle=-7 \mathrm{~m} / \mathrm{s},\left\langle\mathrm{V}_{r}\right\rangle=3.88 \mathrm{~m} / \mathrm{s},\left\langle\mathrm{V}_{\text {obs }}\right\rangle=$ $-4.36 \mathrm{~m} / \mathrm{s}$, and $\left\langle\mathrm{W}_{\mathrm{obs}}\right\rangle=-6.3 \mathrm{~m} / \mathrm{s}$. However, some data points in Figure 5 having apparently unrealistic $\alpha$ values with large signal-to-noise ratios may be attributed to the spatially distinctive characteristics of the vertical air motion and precipitation which occur in the vertical and oblique radar volumes, respectively. For example, the data point with $\alpha$ value of -2.9 

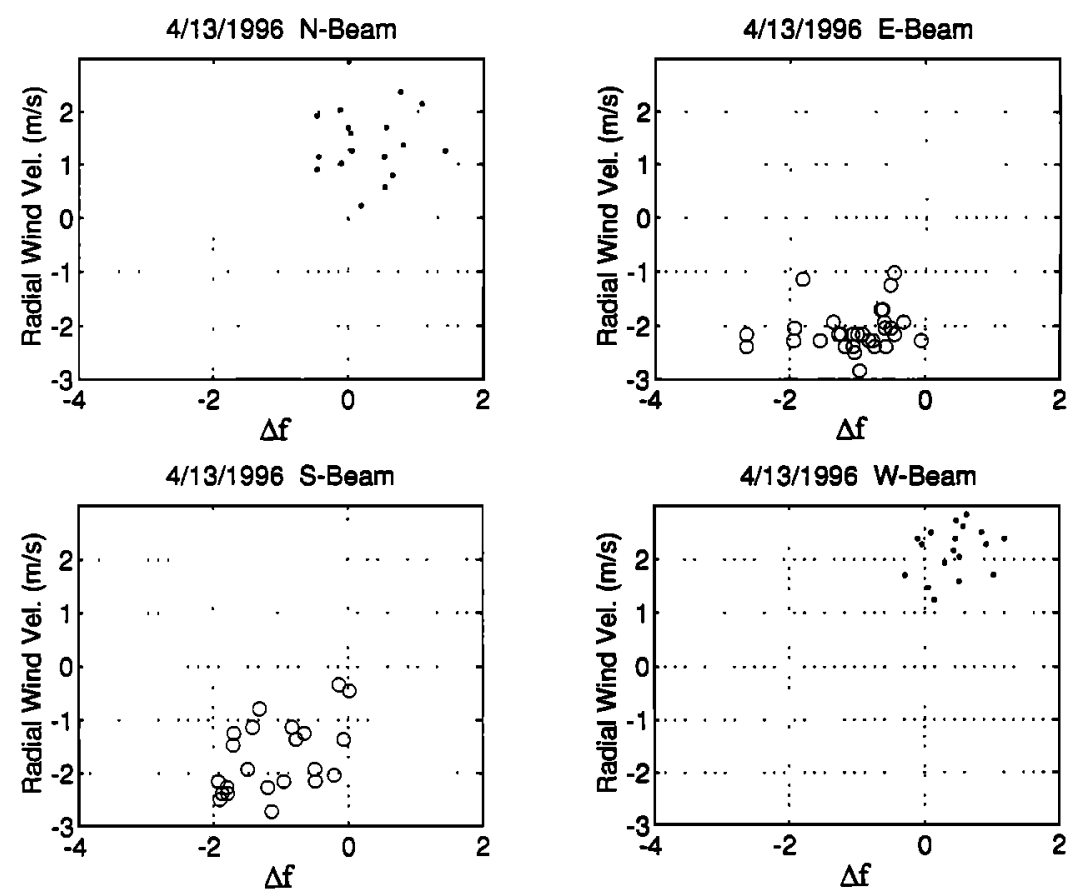

Figure 6. Scatter diagrams of $\Delta f$ versus oblique radial wind velocities at different incident directions, where $\Delta f$ is defined as the discrepancy of the oblique Doppler frequency shift of hydrometeor between observation and theoretical values under the frozen-in assumption. The data points marked with circles (dots) represent cases in which the horizontal wind direction is in the same direction as (opposite direction of) the oblique radar beam.

occurring at the height of $4.2 \mathrm{~km}$ is one of the cases. Moreover, it might be expected that there would be appreciable changes in vertical and horizontal wind speeds and precipitation over the period of a few minutes, especially for the precipitating environment with intense convective activities. These temporal variations of wind speed and precipitation would also lead to the large bias in the estimation of $\alpha$ if the period of taking and processing radar data is not short enough. This is the reason why the interval of observation is less than a minute in this experiment. In view of the fact that several possible factors will cause enormously large deviation of the calculated a values from $\alpha=1$, the interpretation of unrealistic $\alpha$ values must be treated with some caution.

Figure 6 shows the scatter diagram of $\Delta f$ (in units of hertz) versus radial wind velocity observed by the oblique radar beams pointed toward four different directions, where $\Delta f$ is calculated by either (5) or (7), depending on the relative situation that the horizontal wind directions are parallel (shown by circles) or opposite (shown by solid dots) to the radar beam directions. As described in section $2, \Delta f$ will be greater or smaller than 0 , depending on the directions and the relative magnitudes of the vertical and oblique radial velocities. As indicated in Figure 6, the data points marked with circles deviate from the value of $\Delta f=0$ systematically, showing that $\alpha \neq 0$ or that the drift velocity of the precipitation particles is not the same as the background wind. Although the data points marked with dots distribute randomly around $\Delta f=0$, it does not mean that the corresponding hydrometeors are moving with the background wind at the same velocity. This is because the magnitudes of $-f_{a} \times \cos \theta$ are comparable to those of $f_{u}$ such that no systematic variation of $f_{a r}$ (or $\Delta f$ ) with radial velocity is seen. It may be concluded from the observational results presented in Figures 5 and 6 that, in general, the precipitation particles are not frozen in the ambient wind.

\section{Concluding Remarks}

In this paper, taking advantage of the capability of simultaneously observing the precipitation particles and the atmospheric refractivity fluctuations with a VHF radar, we develop a method of examining the wind-driven property of hydrometeor using the Chung-Li VHF radar. In order to comply with the 
requirement made in the proposed method, the vertically and obliquely pointed radar beams are operated simultaneously. Observational result shows that, on average, the efficiency of the ambient atmosphere driving the precipitation particles to drift with the background wind is about 0.83 . This feature implies that the estimations of drop-size distribution and terminal velocity of the precipitation particles associated with strong background wind will be estimated inaccurately if the conventional methods are employed and the wind-driven effect on the movement of hydrometeors is not taken into consideration. In addition, we also note that the spatial distribution of precipitation particles aloft may be considerably inhomogeneous, even in the area with horizontal extent as small as a few hundred meters. This property will increase the difficulty of investigating the characteristics of precipitation by using the radar beams with large tilted angle. Because the frozen-in assumption is a vital one in applying $\mathrm{VHF} / \mathrm{UHF}$ radar (or wind profiler) to observe the characteristics of precipitation aloft, more efforts need to be made to clarify the wind-driven property of hydrometeor. For example, what is the difference of the response to the drag force exerted by the ambient wind between solid and liquid precipitation particles? Is there any seasonal variation for the wind-driven characteristics of precipitation particles? How can one remove the imperfect wind-driven effect on the precipitation Doppler spectrum to obtain a more accurate drop-size distribution of precipitation? We hope that the answers to these questions can be achieved in the near future.

Acknowledgments. This work was partially supported by the National Science Council of Taiwan (R.O.C.) under grant NSC85-NSPO-ECP-008-01. One of the authors (Y.H.C.) would like to express his sincere appreciation to S. K. Avery for her generous hospitality during his visit to CIRES of University of Colorado.

\section{References}

Atlas, D., R. C. Srivastava, and R. S. Sekon, Doppler radar characteristics of precipitation at vertical incidence, $R e v$. Geophys., 11, 1-35, 1973.

Battan, L. J., Radar Observations of the Atmosphere, 323 pp., Univ. of Chicago Press, Chicago, Ill., 1973.

Beard, K., and A. J. Heymsfield, Terminal velocity adjustment for plate-like crystals and graupel, J. Atmos. Sci., 45, 3515-3518, 1988.

Chu, Y. H., and C. H. Lin, The severe depletion of turbulent echo power in precipitation observed using the
Chung-Li VHF Doppler radar, Radio Sci., 29, 1311-1320, 1994.

Chu, Y. H., L. P. Chian, and C. H. Liu, The investigation of the atmospheric precipitations by using the Chung-Li VHF radar, Radio Sci., 26, 717-729, 1991.

Currier, P. E., S. K. Avery, B. B. Balsley, K. S. Gage, and W. L. Ecklund, Use of two wind profilers in the estimation of raindrop size distribution, Geophys. Res. Lett., 19, 1017-1020, 1992.

Foote, G. B., and P. S. du Toit, Terminal velocity of raindrops aloft, J. Appl. Meteorol., 8, 585-591, 1969.

Lin, Y. L., R. D. Farley, and H. D. Orville, Bulk parameterization of the snow field in a cloud model, J. Clim. Appl. Meteorol., 22, 1065-1092, 1983.

Marshall, J. S., and W. M. Palmer, The distribution of raindrops with size, J. Meteorol., 5, 165-166, 1948.

Rajopadhyaya, D. K., P. T. May, and R. A. Vincent, A general approach to the retrieval of raindrop size distributions from wind profiler Doppler spectra: Modeling results, J. Atmos. Oceanic Technol., 10, 710-717, 1993.

Rogers, R. R., A Short Course in Cloud Physics, 227 pp., Pergamon, Tarrytown, N. Y., 1976.

Rottger, J., et al., The Chung-Li VHF radar: Technical layout and a summary of initial results, Radio Sci., 25, 487-502, 1990.

Sue, C. L., An investigation of characteristics of frontal precipitation in the Taiwan area by using the Chung-Li VHF radar (in Chinese), Master's thesis, Natl. Cent. Univ., Chung-Li, Taiwan, 1996.

Ulbrich, C. W., Natural variations in the analytical form of the raindrop size distribution, J. Clim. Appl. Meteorol., 22, 1764-1775, 1983.

Wakasugi, K., A. Mizutani, and M. Matzuno, A direct method for deriving drop-size distribution and vertical air velocities from VHF Doppler radar spectra, J. Atmos. Oceanic Technol., 3, 623-629, 1986.

Woodman, R. F., and Y. H. Chu, Aspect sensitivity measurements of VHF backscatter made with the Chung-Li radar: Plausible mechanism, Radio Sci., 24, 113-126, 1989.

Zrnic, D. S., N. Balakrishnan, C. L. Ziegler, V. N. Bringi, K. Aydin, and T. Matejka, Polarimetric signatures in the stratiform region of a mesoscale convective system, J. Appl. Meteorol., 32, 678-693, 1993.

Y.-H. Chu and T. H. Lin, Institute of Space Science, National Central University, Chung-Li 32054, Taiwan. (e-mail: t272665@twncu865.ncu.edu.tw)

T.-Y. Chen, Institute of Electronics, National Chiao Tung University, Hsin-Chu 30077, Taiwan. (e-mail: t272665@ twncu865.ncu.edu.tw)

(Received August 7, 1996; revised December 12, 1996; accepted January 7, 1997.) 